\title{
Design and fabrication of a fixed device of sedenion and circular ultrasonic sensor
}

\author{
Saike Yang \\ School of North China Electric Power University, Baoding,China
}

1511788086@qq.com

Keywords: Partial discharge, fixed device, ultrasonic array sensor

\begin{abstract}
Partial discharge of electrical equipment will seriously affect the equipment insulation. Ultrasonic array sensor is a mature tool which is used to detect the partial discharge. We design a fixed device of sedenion and circular ultrasonic array sensor, that can meet the precision demands and fixed demands. The device with a suction cup fixtures we designed is easily to carry and use and it's stable.
\end{abstract}

\section{Introduction}

In the electric power system, partial discharge will often happen on electrical equipments such as transformers, which will seriously affect the equipment insulation and make the insulation strength of the equipment decrease rapidly. Ultrasonic array sensor is a common device to inspect the partial discharge, but nowadays the design of fixed device of ultrasonic array sensor is not mature. Therefore, we try to design a fixed device of sedenion and circular ultrasonic array sensor, which can meet the needs of all kinds of electrical equipments and easy to use.

\section{Introduction of the partial discharge}

In the electrical equipments, especially the high-tension equipments, when the electric field generated by the applied voltage in the electrical equipments is sufficient to cause the partial area to discharge, but there is not a fixed discharge channel in the discharging area, it will generate partial discharge.

In the solid dielectric which has gas or liquid, the part of gas or liquid discharge when the local field of gas or liquid reaches its breakdown strength. Partial discharge is usually due to the concentrated discharge of the interior and surface of the insulator. Generally, the discharge represent that the pulse duration is less than 1 us.

Every time partial discharge will have some impact on the insulation medium. The lighter will have little impact on the power equipment insulation. The insulation strength will decrease slowly. But a stronger partial discharge will make the insulation strength decrease quickly. It is an important factor to damage the high-tension electrical equipment insulation. Therefore, the inspection and maintenance of the partial discharge is necessary for the safe operation of the high-tension electrical equipments.

\section{Design of a fixed device of ultrasonic array sensor}

Ultrasonic array sensor is a kind of important equipment to inspect the source of partial discharge. But now we lack the mature fixed device of ultrasonic array sensor. In view of the existing technical problems, we will provide a fixed device of ultrasonic array sensor with a low cost, high reliability, high safety and high inspection accuracy for partial discharge source.

\section{Introduction of the basic structure}

Our design consists of a handle, a base and a suction cup fixing device. As is shown in the following images, figure 1 is the design of the upward view, figure 2 is the cross-section. 


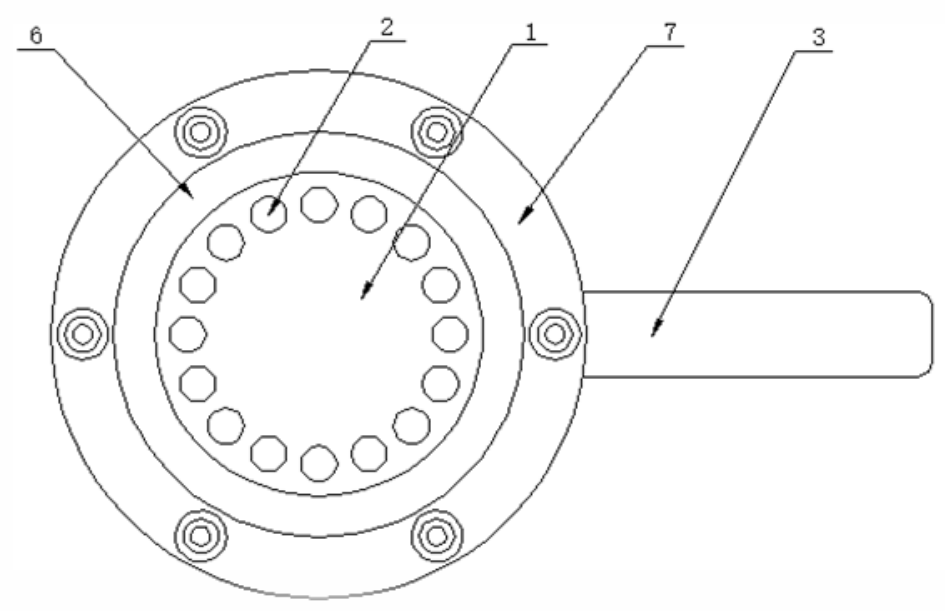

Figure 1

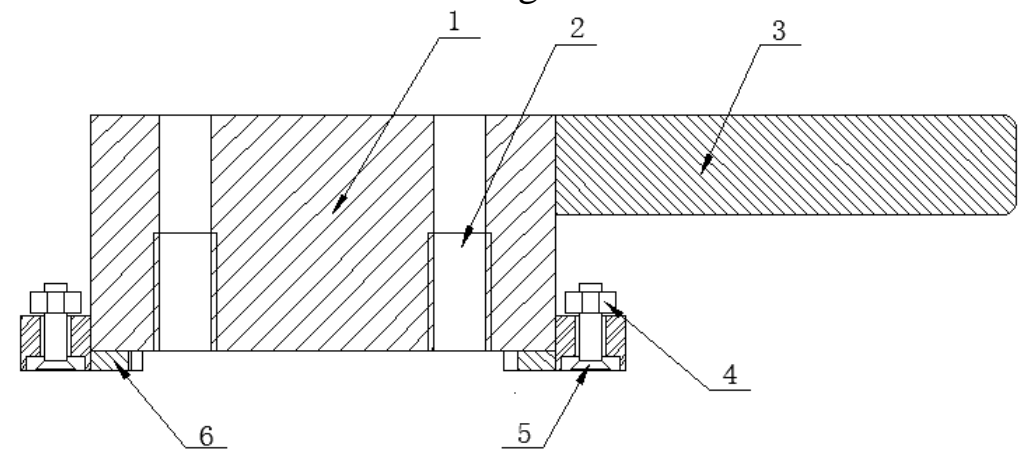

Figure 2

1 base 2 hole 3 handle 4 suction cup fixed nut 5 suction cup 6 magnetic pad 7 suction cup fixing device

This design consists of four parts as base, handle, magnetic pad and suction cup fixing device. Among them, the base is provided with 16 mounting hole, which are installed into a circular array and used for the placement of sensor array. Each hole with inner screw thread is used to fix ultrasonic array sensor. The handle connected with the base is mainly for the convenience of use and carrying. Magnetic pad and suction cup fixing device can be used to fix the device to the electrical equipment. For the equipment of ferromagnetic material, it can be directly adsorbed to the above with the magnetic force of the pad. There are some small holes leading the sensor array wire around the magnetic pad. For non ferromagnetic material, it can be fixed to the electrical equipment with suction cup fixing device. Suction cup fixing device has small holes to lead the sensor array wire. There are evenly distributed with 6 suction cups under the suction cup fixing device, which are used to fix this device to the electrical equipment. At the same time, suction cup fixing device is removable. The exterior of magnetic pad and the interior of suction cup fixing device have screw thread to fix the suction cup fixing device. When we don't need the suction cup fixing device, it can be removed. Figure 3 is the cross-section of the device without the suction cup fixing device. Figure 4 is the cross-section of the suction cup fixing device. 


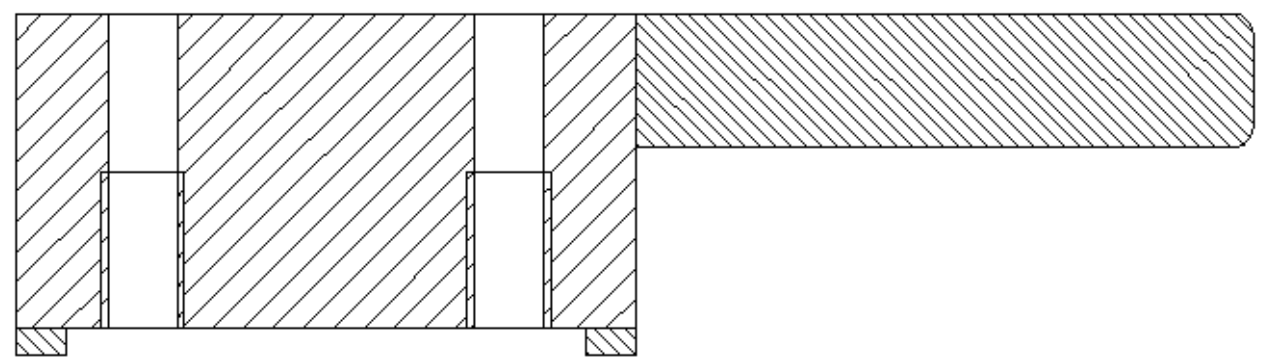

Figure 3

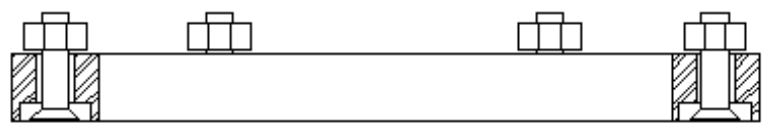

Figure 4

\section{Material introduction of design}

The equipment's base and handle are made of aviation aluminum. Aviation aluminum is a kind of Aluminum Alloy. Main components are: aluminum, copper, zinc, magnesium, silicon and so on. It performs well because of its resistance to oil, acid, alkali, salt and other chemical reagents. It can tolerant organic solvent dissolution and has good chemical properties and molding. The products take the advantages of smooth surface, good abrasion resistance, easily processing and can be used for the secondary operation with machinery and bonding method. It weights light and is easy to carry. Even can adapt to the changes of the external environment, day and night temperature and humidity conditions, the acid, alkali and salt corrosion and other pollution from the bad weather and air pollution, which is suitable as the material of the fixed device of sensor. The suction cup adopts fluorine rubber suction cup. Fluorine rubber has excellent heat resistance, oxidation resistance, oil resistance and aging resistance, and the price is cheap. It is suitable for connecting part of the device and non ferromagnetic materials.

\section{Usage method of the device}

Firstly, make the 16 ultrasonic array sensor fix to the device through the screw, to ensure tightened, ensure they won't drop automatically during the movement or the installation.

Secondly, whether you need to use suction cup fixing device depends on the material selection of the different electrical equipments. If you need the device, first you should install the suction cup fixing device.

Take the lead wire of the ultrasonic array sensor through the lead hole to connect with the receiving device.

It can be directly adsorbed to the relative electrical equipment with the magnetic pad or suction cup when used. In the replacement of detection equipment, as long as it is removed from the original equipment and then install or remove the suction cup fixing device. It can be install on the other new equipment.

\section{Advantage of design}

(1) The suction cup can be firmly fixed in the fixing device. There is no chance to change the relative position and affect the measurement accuracy. 
(2) The excellent usage of the suction cup fixing device. Non ferromagnetic materials can also be used.

(3) Take the method of sedenion and circular array. The measurement accuracy is higher.[1]

\section{Summary}

For the electrical equipment, partial discharge phenomena will produce great insulation effect. The detection of the ultrasonic array sensor to the partial discharge has been more mature. The fixed device of sedenion and circular ultrasonic array sensor designed by us can fix the 16 ultrasonic array sensors to the electrical equipment well. Not only ensure the measurement accuracy, but also meet the need of low cost and high reliability.

\section{References}

[1]Qing Xie, Shuyi Cheng, Fangcheng Lv, etc. Using the improved circular ultrasonic array sensor PD location in oil[J]. High-tension technology.2013,39(5). 